\title{
Restoring chemo-sensitivity to temozolomide via targeted inhibition of poly (ADP-ribose) polymerase-1 by naringin in glioblastoma
}

\author{
Vanishree Rao ${ }^{1}$ - Sri Pragnya Cheruku ${ }^{1}$ Suman Manandhar ${ }^{1}$ - R. J. A. Vibhavari ${ }^{1} \cdot$ Krishnadas Nandakumar $^{1}$. \\ C. Mallikarjuna Rao ${ }^{1} \cdot$ V. Ravichandiran ${ }^{2} \cdot$ Nitesh Kumar $^{1,2}$ (])
}

Received: 11 March 2021 / Accepted: 12 May 2021 / Published online: 28 May 2021

(c) The Author(s) 2021

\begin{abstract}
Inclining mortality with a constant plummet in the survival rates associated with glioblastoma still stands as an inveterate predicament. The only promising therapy with temozolomide (TMZ) is now side-lined due to escalated resistance mediated by Poly (ADP-ribose) Polymerase-1 (PARP-1). In the light of this, the very study was designed to evaluate the potential of an active phyto component named naringin, in inhibiting PARP-1, using in silico and in vitro methods. Under in silico settings, inhibitor bound crystal structure of PARP-1, i.e., 4UND was retrieved and molecular docking studies were performed against naringin using Schrodinger software. In vitro cytotoxicity and apoptotic detection assay were performed using C6 glioma cells. Docking studies revealed high affinity and low binding energy at the inhibition site with good stability. An increase in cytotoxicity to C6 cells was observed with TMZ and naringin combination when compared to TMZ alone. Isobologram plot confirmed the synergistic effect of the drug combination. A significant increase in the number of apoptotic cells with combination drugs, as evaluated by acridine orange and ethidium bromide staining reassured the reversal of resistance. In conclusion, chemosensitivity to TMZ was restored by successful inhibition of PARP-1 using naringin and the drug combination was hence proven effective in reversing TMZ resistance.
\end{abstract}

Keywords Glioblastoma $\cdot$ Temozolomide $\cdot$ Poly (ADP-ribose) polymerase- $1 \cdot$ Naringin $\cdot$ Chemoresistance

\section{Introduction}

Being the third most baleful cancer worldwide, glioblastoma multiforme (GBM) is ill-famed for its fatality (Goldsmith and Carter 1974; Cloughesy et al. 2014). The standard treatment options available in managing this menacing tumor include; surgery, radiotherapy and chemotherapy. In this era, temozolomide (TMZ) is the only first-line chemotherapeutic choice in GBM patients (Newlands et al. 1997). Temozolomide was ratified by FDA in the year 2005, as an orally acting DNA methylating drug for the management of glioblastoma. This prodrug, upon activation, forms MTIC

Nitesh Kumar

niteshkumar43@gmail.com

1 Department of Pharmacology, Manipal College of Pharmaceutical Sciences, Manipal Academy of Higher Education, Manipal, Karnataka 576104, India

2 Department of Pharmacology and Toxicology, National Institute of Pharmaceutical Education and Research, Vaishali, Hajipur, Bihar 844102, India (5-(3-dimethyl-1-triazenyl) imidazole-4-carboxamide), which is severed by water molecule engendering highly active methyl diazonium cation. Post-haste, this methyl moiety is transferred from the cation to DNA strands at O6-guanine, N3- guanine and N7- adenine loci. Due to the interference of these methyl adducts, the normal DNA replication and base-pairing process is curtailed, resulting in cell cycle arrest at the G2/M phase. Futile cycles of cell cycle arrest cease the tumor cell division process and cause tumor cell death (Zhang et al. 2012).

Chemotherapy with temozolomide emerged to be a promising approach with the snag being rapid development of resistance to chronic chemotherapy in most of the patients (Lee 2016). Despite fiendish temozolomide treatment, the survival rates of glioma patients keep plummeting. The rapid development of resistance to temozolomide treatment in patients is the core reason for the lower survival rates. Significant DNA repair pathways, in particular, direct repairing by Methyl Guanine Methyl Transferases (MGMT), indirect repairing systems like base excision repair (BER) and missmatch repair (MMR) have been hypothesized for mediating 
the resistance (Johannessen and Bjerkvig 2012; Messaoudi et al. 2015). BER is an internal cell repair system that plays a salient role in nullifying the detriment triggered by a wide range of oxidizing factors, ionizing radiation or methylating/alkylating drugs (Trivedi et al. 2005). This system is inclusive of copious variable enzymes and proteins. One of them, Poly [ADP-ribose] polymerase 1 (PARP-1) is majorly expressed in situations of DNA damage. Upon binding to the corrupted DNA, PARP-1 initiates the synthesis of poly (ADP-ribose), which facilitates the assembly of BER repair proteins (ligase, DNA polymerase, $\mathrm{XRCC} 1, \mathrm{FEN}-1$ ) thereby establishing repair mechanisms (Gupta et al. 2019; Higuchi et al. 2020). Since the binding of PARP-1 activates the downstream DNA repair activities, this becomes an ace target. At present, selective/non-selective inhibitors of PARP-1 like rucaparib, olaparib and niraparib have been approved for ovarian cancer. However, due to extensive systemic side effects and poor blood-brain barrier penetrability, their use in glioma remains questionable. (Franzese et al. 2019).

Bioflavonoids constituting a ubiquitous group of polyphenolic compounds are currently the trending trial treatment entities in various fields (Nijveldt et al. 2001). Naringin (IUPAC name: 7-[[2-O-(6-Deoxy- $\alpha$-L-mannopyranosyl)$\beta$-D-glucopyranosyl]oxy]-2,3-dihydro-5-hydroxy-2-(4hydroxyphenyl)-4H-1-benzopyran-4-one), a flavanone-7-Oglycoside has been a popular contender in the field of therapeutics because of its potent antioxidant, anti-cancer, anti-apoptotic, lipid-lowering and metal chelating activities (Raja Kumar et al. 2019; Salehi et al. 2019). Rich natural occurrence of naringin is seen in citrus and grapefruits where it superintends for the bitterness of these fruits (Jourdan et al. 1985). Recent studies using cancer cell lines postulated that anti-cancer/anti-apoptotic activities by naringin might be due to the potential inhibition of copious members of the PARP protein family (Su et al. 2017). Since PARP-1 is involved in mediating TMZ resistance, naringin could prevent or reverse the chemoresistance by binding to PARP-1 thereby inhibiting the activation. With this knowledge, the present study was designed to explore the ability of naringin to inhibit PARP-1 protein, in silico and in vitro, in order to reverse the development of chemoresistance to temozolomide in GBM.

\section{Materials and methods}

\section{In silico methodology}

\section{Molecular docking calculations}

Computational docking/simulation studies were performed using Schrodinger software. Commercial Maestro software, version 11.8 (OPLS3e force field) developed by
Schrodinger was used for all the simulation studies. This software was used on hp computer with the operating system Linux Ubuntu 18.04.1 Long Term Support. All the desired programs such as protein preparation, ligand preparation, molecular docking, MMGBSA (Molecular MechanicsGeneralized Born Surface Area) and molecular dynamics were performed in an hp system with an inbuilt Intel Haswell graphics card, Intel Core i3 processor and 4 GigaByte Random-access memory (RAM).

Crystal structure of PARP-1 (Protein Data Bank ID: 4UND, crystallographic precision of the PDB: $2.20 \AA$ ), with bound ligand, Talazoparib (8S,9R)-5-fluoro-8-(4fluorophenyl)-9-(1-methyl-1H-1,2,4-triazol-5-yl)-2,7,8,9tetrahydro-3H-pyrido[4,3,2-de]phthalazin-3-one) which is an approved anticancer drug, was retrieved from RCSB Protein data bank (Berman et al. 2000). The protein structure was prepared for further docking and dynamic studies by using protein preparation panel which included processing, review, modify and refinement modules. Water molecules beyond $3 \AA$ were removed during the preparation. To generate the grid around the receptor for docking, receptor grid generation, an inbuilt panel named GLIDE was used. The grid was generated at the binding site of talazoparib (Rathi et al. 2019). The grid box of size $10 * 10 * 10 \AA$ was generated at the binding site of the inbound ligand, talazoparib.

The structure of naringin (Molecular formula: C27H32O14) was initially retrieved from the ZINC 15 database in the MOL-2 lowest energy format (ZINC ID: ZINC8143604). Later, the structure was imported into Schrodinger's Maestro software and Ligand preparation was done using the "LIGPREP" panel (Rathi et al. 2019).

After protein and ligand preparation, molecular docking was performed using the glide module of Maestro. Initially, docking was performed at standard precision (SP) mode, followed by extra precision (XP) mode. This step was performed to obtain a ligand docking score (Elokely and Doerksen 2013).

\section{Free ligand binding energy calculation by Maestro (MM-GBSA)}

The binding and strain energy required during the binding of the target protein listed above with naringin was given by the PRIME module. After performing ligand docking, XP files corresponding to each naringin and PARP-1 docking was used to conduct MM-GBSA (Ignjatović et al. 2016).

\section{Molecular dynamic (MD) simulations}

The MD simulation assessment was implemented on PARP-1 and naringin, using the XP docking file via DESMOND panel of maestro for a period of $20 \mathrm{~ns}$. A total of three steps were involved in this process namely, system 
builder, minimization and MD simulation. For the XP docked complex of 4UND and naringin, a predefined simple point charge model (SPC) was used for the creation of an orthorhombic boundary. Three positive charges and one negative charge on the model was neutralized by the addition of one sodium and three chloride ions. A constant temperature of $300 \mathrm{~K}$ and 1 bar pressure was maintained throughout the simulation under the isothermal-isobaric (NPT) ensemble. Parameters like Root Mean Square Deviation (RMSD), protein-ligand contact timeline and covalent/ non-covalent interactions were analyzed (Ivanova et al. 2018).

\section{In vitro methodology}

C6 cell line (rat origin) was used in this study and it was procured from NCCS (National Centre for Cell Science), Pune. The cell line was cultured under sterile conditions in cell culture lab, MCOPS, MAHE, Manipal and maintained in Dulbecco's Modified Eagle Medium (Invitrogen) with $10 \%$ Fetal Bovine Serum (Invitrogen), $4 \mathrm{mM}$ glutamine, $100 \mathrm{U} / \mathrm{ml}$ penicillin and $100 \mu \mathrm{g} / \mathrm{ml}$ streptomycin. The culture medium was changed daily and $5 \mathrm{ml}$ of media was added after thawing the aliquoted media to room temperature. Cells were maintained at $37{ }^{\circ} \mathrm{C}$ with $5 \% \mathrm{CO} 2$ supplement, in a humidified incubator as recommended by the American Type Culture Collection (ATCC; Rockville, MD, USA).

\section{Cytotoxicity assay}

SRB (Sulforhodamine B) colorimetric assay was performed to assess the cytotoxicity of TMZ (Sigma-Aldrich, India) and naringin. Naringin was procured from TCI chemicals, Bangalore and $100 \mathrm{mM}$ stock solution of this was prepared in $1 \mathrm{ml}$ of DMSO (Dimethyl sulfoxide). C6 cells grown on T25 (tissue culture treated) flasks were detached from the surface using trypsin-EDTA solution. The cell suspension obtained was centrifuged for $3 \mathrm{~min}$ at 1500 RPM. Based on cell count, cell suspension was prepared and 5000 cells/ well were seeded into a 96-well plate. After $24 \mathrm{~h}$ of seeding, cells were treated with 7 serial doses. Treatment with temozolomide and naringin alone was carried out with following doses: $500 \mu \mathrm{M}, 250 \mu \mathrm{M}, 125 \mu \mathrm{M}, 62.5 \mu \mathrm{M}$, $31.25 \mu \mathrm{M}, 15.63 \mu \mathrm{M}$ and $7.810 \mu \mathrm{M}$. For combination study, recommended equipotency ratio of $1: 1$ was used. Both the drugs were added to the same well in the order $250 \mu \mathrm{M}+250 \mu \mathrm{M}, 125 \mu \mathrm{M}+125 \mu \mathrm{M}, 62.5 \mu \mathrm{M}+62.5 \mu \mathrm{M}$, $31.25 \mu \mathrm{M}+31.25 \mu \mathrm{M}, \quad 15.63 \mu \mathrm{M}+15.63 \mu \mathrm{M}$, $7.810 \mu \mathrm{M}+7.810 \mu \mathrm{M}$ and $3.905 \mu \mathrm{M}+3.905 \mu \mathrm{M}$. After $48 \mathrm{~h}$ of incubation at room temperature, SRB assay was performed and plate reading was taken at $540 \mathrm{~nm}$ (Aslantürk 2018). Obtained data were used to calculate the $\mathrm{IC}_{50}$ values of each treatment. $\mathrm{IC}_{50}$ values were fed into "COMPUSYN" (V.1) software in order to determine combination index and type of effect showed by drug combination (antagonism/ synergism/ potentiation) (Zhang et al. 2016).

\section{Computerized simulation using COMPUSYN software}

CompuSyn was downloaded upon free registration from its official website www.combosyn.com. "User's Guide for CompuSyn" was referred for understanding the automated system of dose-effect analysis of simulation (Chou and Martin 2005). The particular dose administered and corresponding "fraction of cells affected" calculated by SRB assay was entered manually at the designated places in CompuSyn software. The report generated carrying all the results was viewed using the internet explorer application (Zhang et al. 2016).

\section{Estimation of apoptosis/necrosis in $\mathrm{C} 6$ cells by Acridine orange/ ethidium bromide (AO/EB) staining}

Six well plates were seeded with C6 cells at $3^{*} 10^{6}$ density. Cells were allowed to attach for $24 \mathrm{~h}$ and upon obtaining the monolayer, cells were pre-treated with naringin with previously calculated $\mathrm{IC}_{50}$ dose and incubated at room temperature. After $24 \mathrm{~h}$, cells were treated with TMZ $\left(\mathrm{IC}_{50}\right.$ dose) alone and in combination and incubated. After incubation for $24 \mathrm{~h}$, cells were washed thrice with freshly prepared phosphate-buffered saline and added with $1 \mathrm{ml}$ of ethanol per well for fixation. After $10 \mathrm{~min}$, freshly prepared AO/EB staining was added to each well ( $1 \mathrm{ml}$ for $10 \mathrm{~min})$. The cells were viewed under an Inverted microscope with fluorescence filters for morphological examination (Tiwari et al. 2016).

\section{Statistical analysis}

Statistical analysis of data was performed using one-way analysis of variance followed by Tukey's post hoc test. Graph pad prism (V.8) software, installed at the institution was used for analysis. Analyzed data are expressed as mean \pm SEM.

\section{Results}

\section{In silico computational docking studies}

\section{Molecular docking score, interactions and binding energy}

Result of XP molecular docking using GLIDE reported a docking score of $-11.648 \mathrm{kcal} / \mathrm{mol}$ with three types of non-covalent interactions (Table 1). Since the co-crystallized inhibitor was bound to chain B of the protein, chain A 
Table 1 Docking details of naringin

\begin{tabular}{lllll}
\hline S. no & Ligand name with ZINC ID & 2D structure \\
\hline 1 & Naringin \\
ZINC8143604
\end{tabular}

ZINC ID and 2D structure of naringin with docking score and binding energy calculated by Schrodinger software

was not included in any of the applications. As illustrated in Table 2, amino acid residues namely Asn767, Ser864, Asp770, Arg878, Glu988 and Gly863 depicted strong hydrogen bond formation with naringin. The hydrogen bond-forming property between a ligand and protein plays a pivotal role in drug designing. In this case, ten hydrogen bonds were established in the catalytic domain. Two prominent hydrophobic contacts between hydrophobic amino acid residues Tyr896, Tyr907 and aromatic rings of naringin further contributed for the stabilization of the complex. Hydrogen bonded protein and ligand interactions mediated by water molecule, also called water bridges were formed at amino acid residues Asp756, Asn767, Ser864, Tyr889, His862 and Gly888. No ionic interactions were noted between naringin and the protein, PARP-1.
In order to evaluate more accurate binding affinity, the XP file obtained was subjected to the Prime MM-GBSA method. The binding energy (MM-GBSA $\Delta \mathrm{G}$ ) calculated for this complex was $-42.766 \mathrm{kcal} / \mathrm{mol}$. In alignment with previous literature, this value was found to be significant (Table 1).

\section{Molecular dynamics}

The in silico investigation was further deepened by performing a molecular dynamic study for $20 \mathrm{~ns}$. Obtained RMSD values were explicated to get more insights on binding stability of docked complex. RMSD values of the PARP-1 (4UND) and naringin were found to be $4.6 \AA$, $2,8 \AA$ respectively (Fig. 1). An initial rise in the RMSD plot of both

Table 2 Protein-ligand interaction diagram

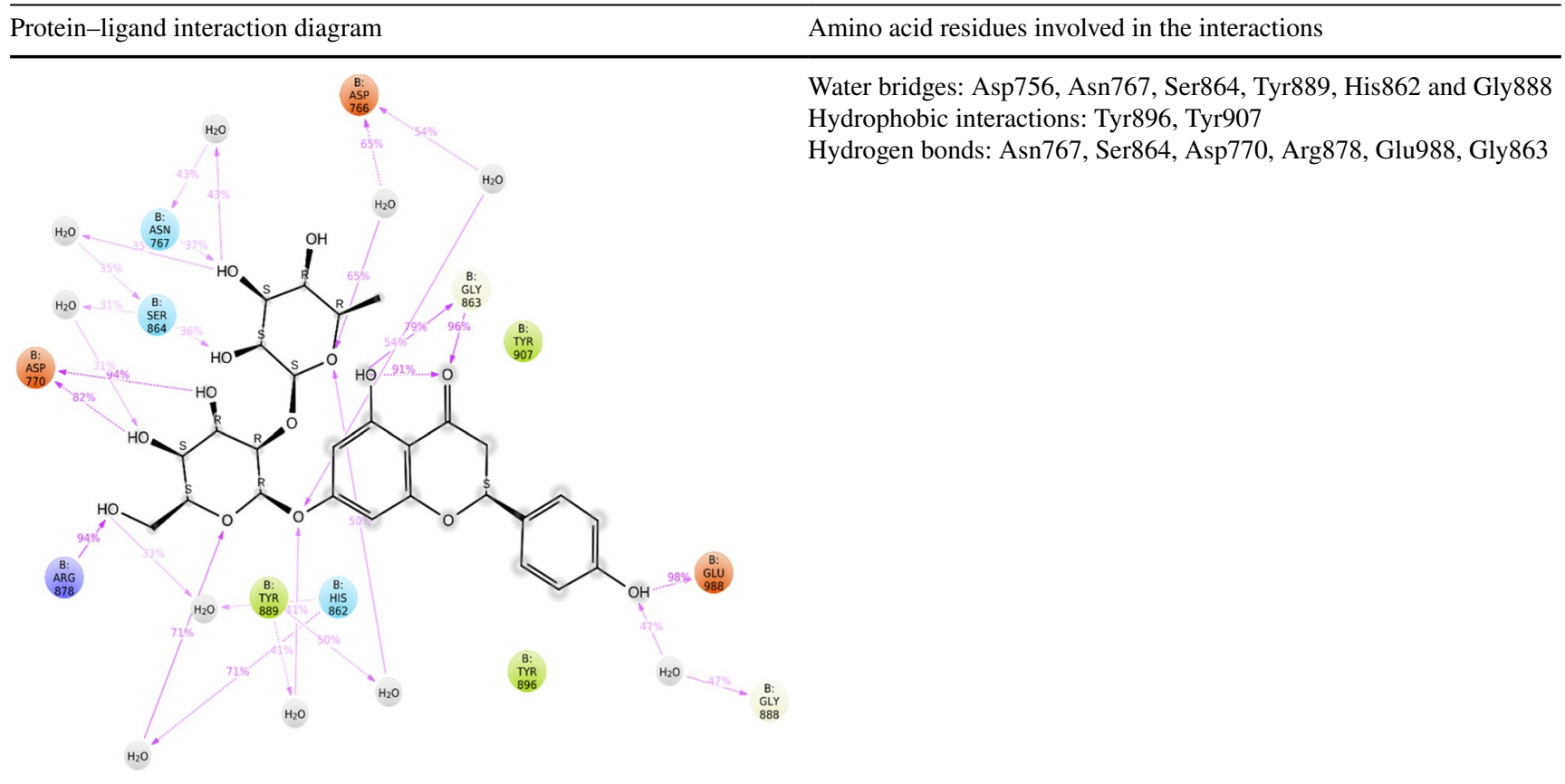

PARP-1 and naringin interaction included multiple hydrogen bonds, hydrophobic bonds and water bridges 


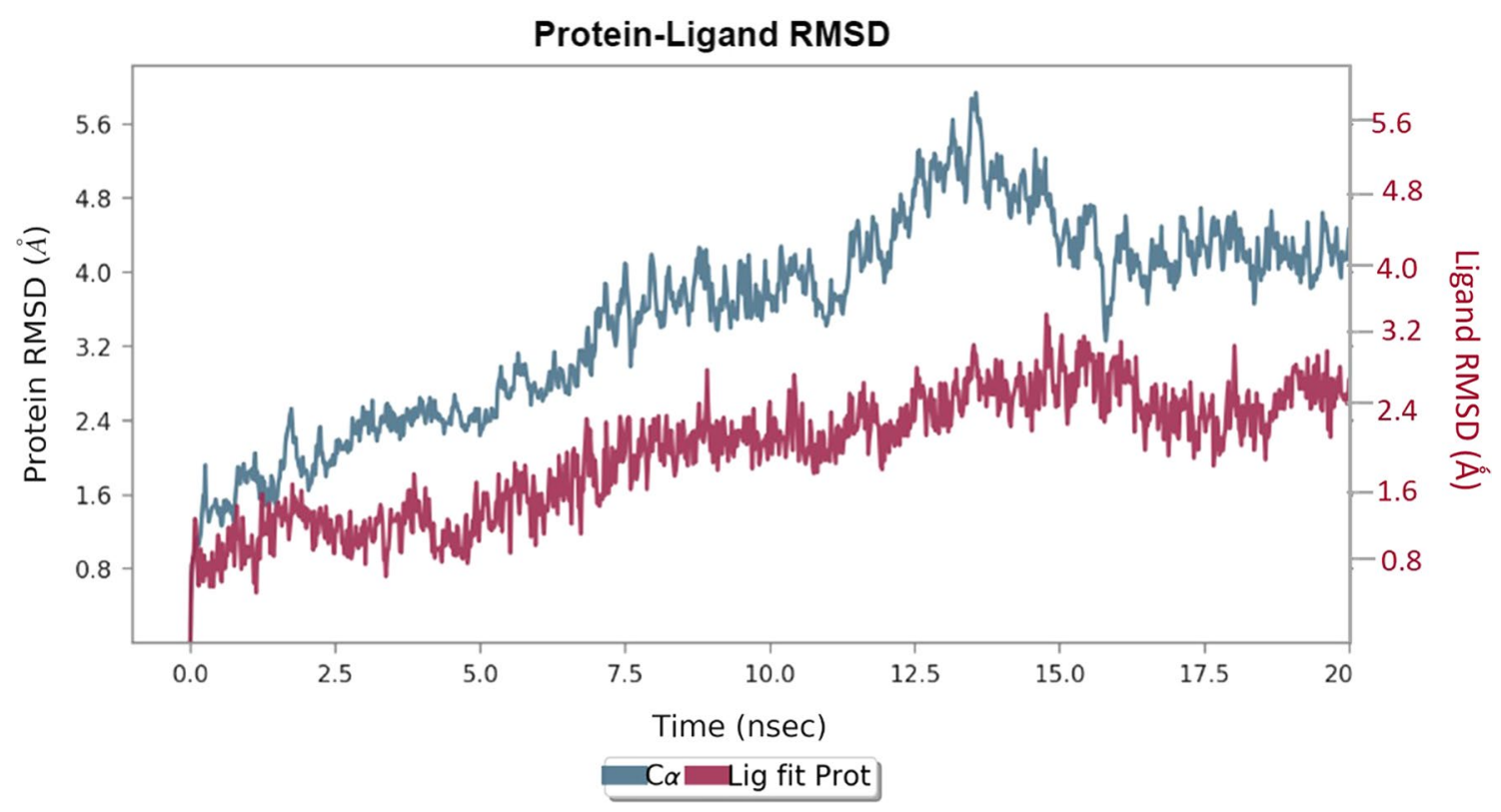

Fig. 1 Protein-ligand root mean square deviation (RMSD) plot. RMSD plot of naringin bound to active inhibitory site at PARP-1 protein (4UND)

ligand and protein was well stabilized after $15 \mathrm{~ns}$ (Fig. 2). The molecular dynamics trajectory showed that, up to $10 \mathrm{~ns}$, amino acid residues like Glu763, Arg865, Gly888, Met890 and Lys903 were important before ligand (naringin) stabilization. Later that, amino acid residues such as; Asn767, Ser864, Asp770, Arg878, Glu988, Gly863, Tyr896, Tyr907, Asp756, Asn767, Ser864, Tyr889 and His862 established strong contacts with the ligand to be the pivotal hotspots for stabilization (Fig. 3).

Figure 4 illustrates the plot of ligand and protein contacts and interaction fraction. The stacked bar charts were normalized over the period of the trajectory. The interaction fraction represents the total period of the simulation for which the specific interaction was maintained. Most of the hydrogen bonds, water bridges or hydrophobic interactions are maintained $>1 \mathrm{~ns}$ implementing that the interactions are stable and strong.

\section{Cell culture-based studies using C 6 glioma cell line}

\section{Cytotoxicity study}

Percentage cell death and $\mathrm{IC}_{50}$ values of treatment groups were calculated via SRB assay. The cytotoxicity study was performed in a dose-dependent manner. Calculated $\mathrm{IC}_{50}$ values of treatment groups such as temozolomide (TMZ), naringin (NRG) and temozolomide plus naringin (TMZ+NRG) were $83.05 \mu \mathrm{g} / \mathrm{ml}, 162.93 \mu \mathrm{g} / \mathrm{ml}$ and $15.50 \mu \mathrm{g} / \mathrm{ml}$ respectively. A decrease in $\mathrm{IC}_{50}$ value was seen in the treatment group receiving drug combination $\mathrm{TMZ}+\mathrm{NRG}$ when compared to individual drug treatments. The percentage of cell death recorded corresponding to each dose is listed in Table 3.

\section{Computerized simulation results}

The final obtained report from CompuSyn included dose-effect curves, median-effect plot, m, Dm and r parameters, fa-CI (fraction affected and Combination index) table, Fa-CI plot, isobologram and summary of the findings (Fig. 4). Complete computer-generated explicit details of the study are given in supplementary material.

All datasets corresponding to each treatment group were initially analyzed for $\mathrm{m}$, $\mathrm{dm}$ and $\mathrm{r}$ values (Table 4). "m" corresponds to the shape of the dose-effect curve (Fig. 4b). Here, curve corresponding to TMZ and NRG treated groups assumed flat sigmoidal shape $(\mathrm{m}>1)$ and $\mathrm{TMZ}+\mathrm{NRG}$ showed sigmoidal shape $(\mathrm{m}<1)$. "dm" values depicted in Table 4, represents median effect (ME) dose $\left(\mathrm{IC}_{50}\right.$ value). The linear correlation coefficient of the ME plot is given as " $r$ " values. This value mainly signifies the conformity of the data entered. All the treatment groups have shown acceptable/good data with $r>0.95$.

\section{The dose-effect plot}

The dose-effect plot (Fig. 4a) explains the cytotoxic effect of each treatment group on the C6 cell line at various doses. NRG treated group shows the least cell death at various doses when compared with TMZ and TMZ + NRG. 

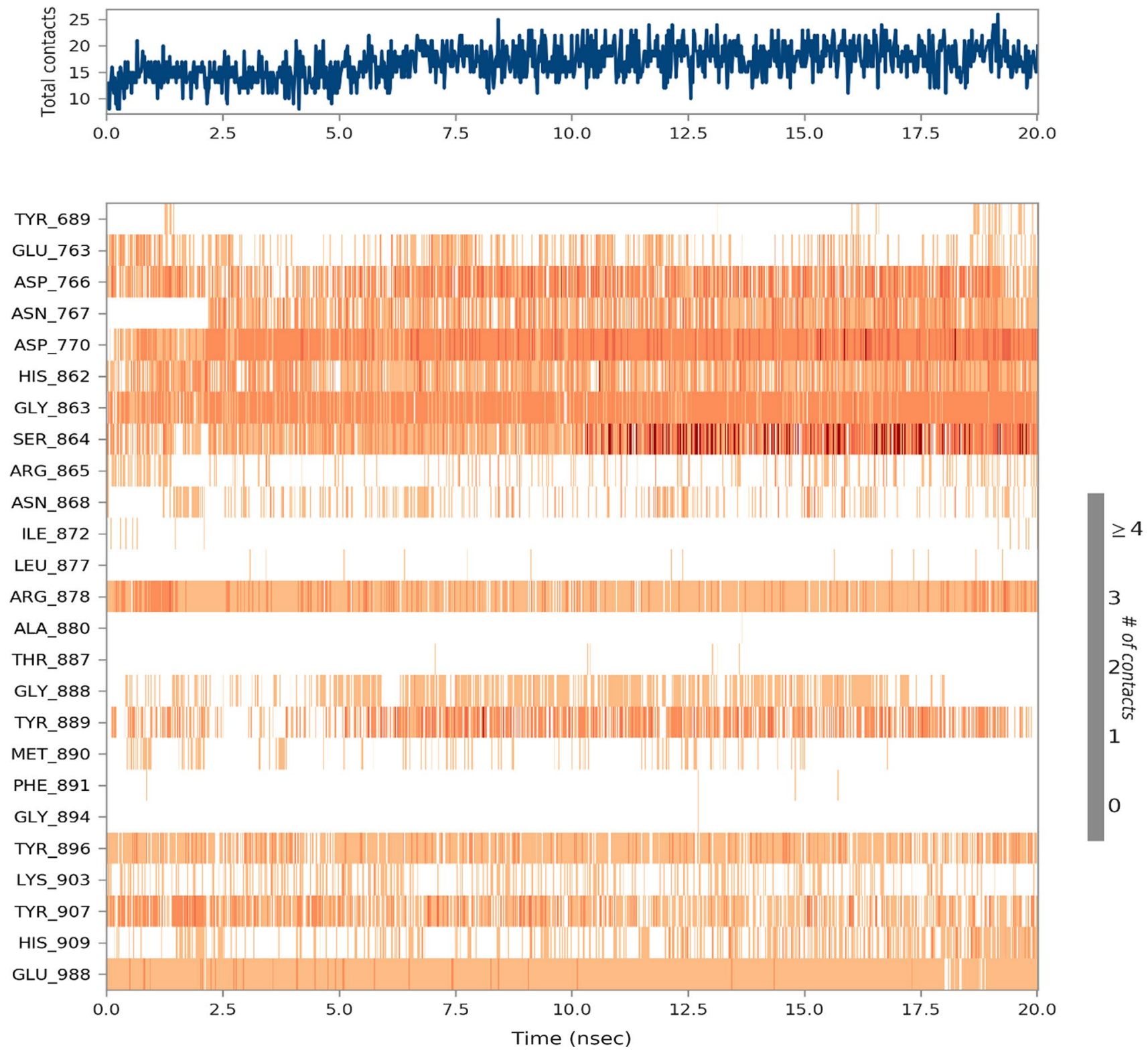

Fig. 2 Protein-ligand contact timeline plot. Total contact timeline plot of naringin bound to active inhibitory site at PARP-1 protein (4UND)

Treatment using a combination of drugs (TMZ + NRG) has shown a rise in response/cell death at given doses in comparison with individual drug-treated groups (Table 3 ).

\section{Combination index}

The combination index (CI) is calculated to understand the type of effect observed when drugs are given in combination. $\mathrm{CI}<1,=1$, and $>1$ indicate synergism, additive effect and antagonism, respectively. In this case, the CI value has come out to be $<1$ at both high and low doses hence proving a synergistic effect (Table 5). The combination index plot (Fig. 4c) depicts the synergistic effect since all the data seven points entered are lying below the margin $(\mathrm{CI}=1)$.

\section{Isobologram plot}

Isobologram of $50 \%, 75 \%$ and $90 \%$ (fa: $0.5,0.75,0.9$ ) are for combination data points are shown in Fig. 4d. In this case, $\mathrm{IC}_{50}, \mathrm{IC}_{75}$ and $\mathrm{IC}_{90}$ showed synergism as the points are lying on the lower left side of the plot. 


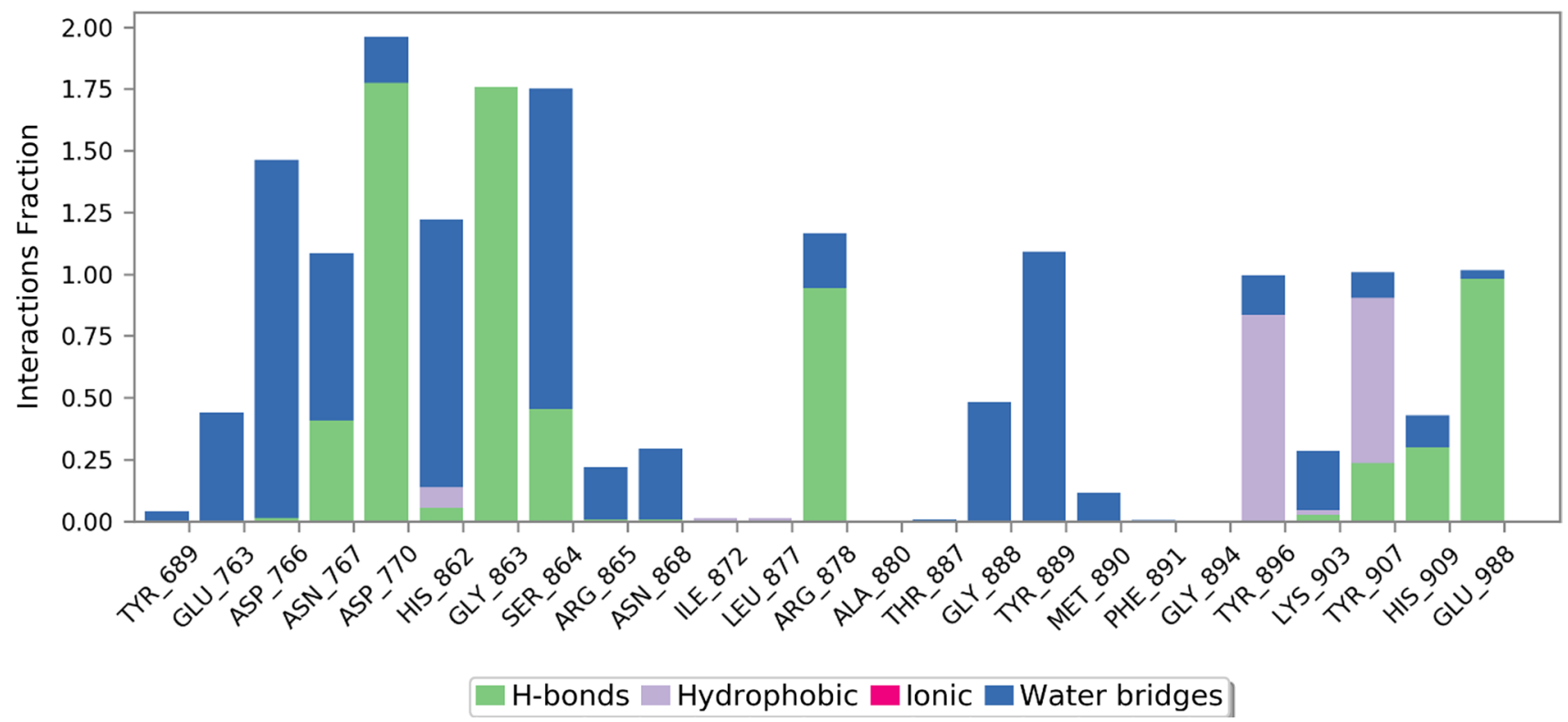

Fig. 3 Protein interaction diagram various types of interactions established at the amino-acid residual sites during protein-ligand simulation
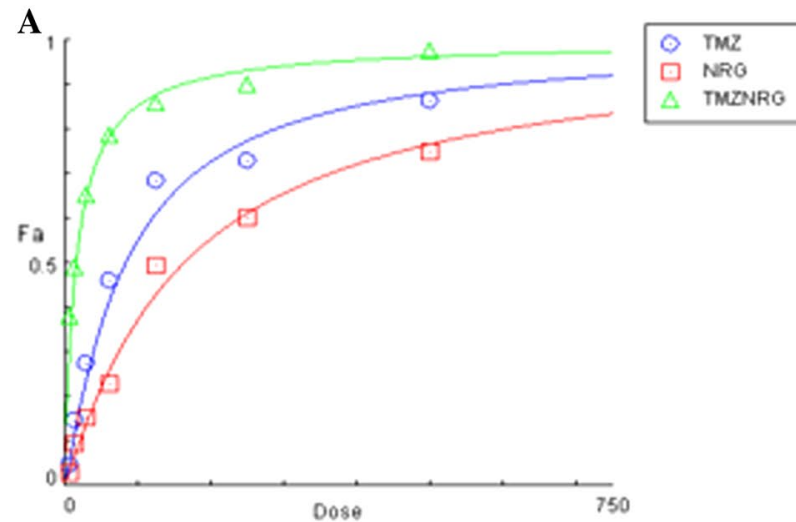

C

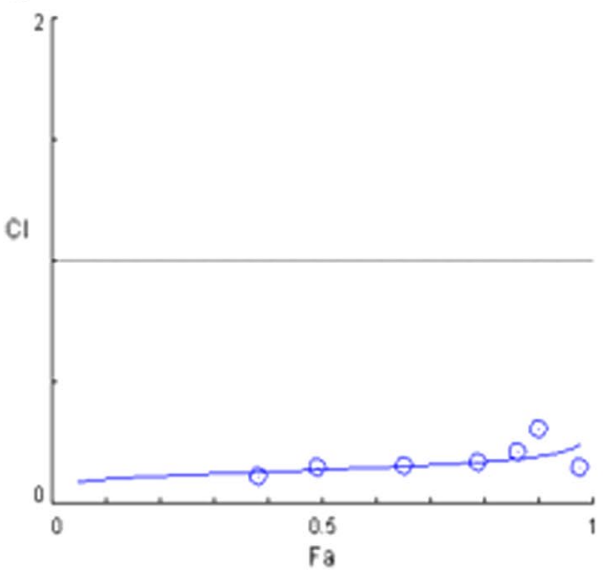

B

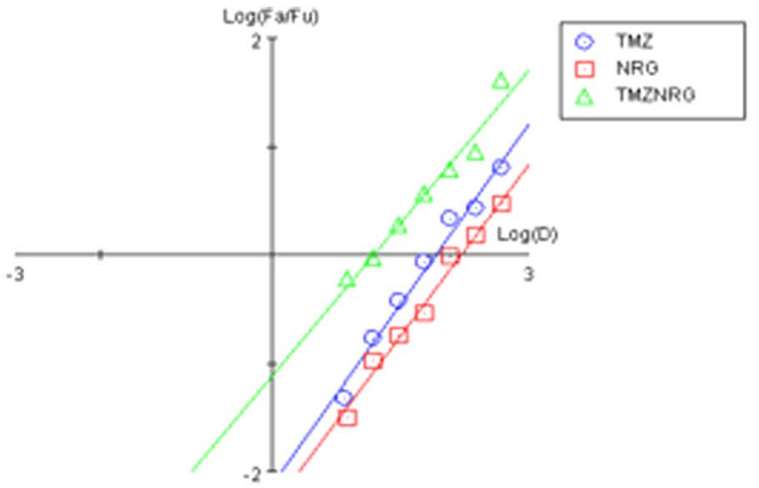

D

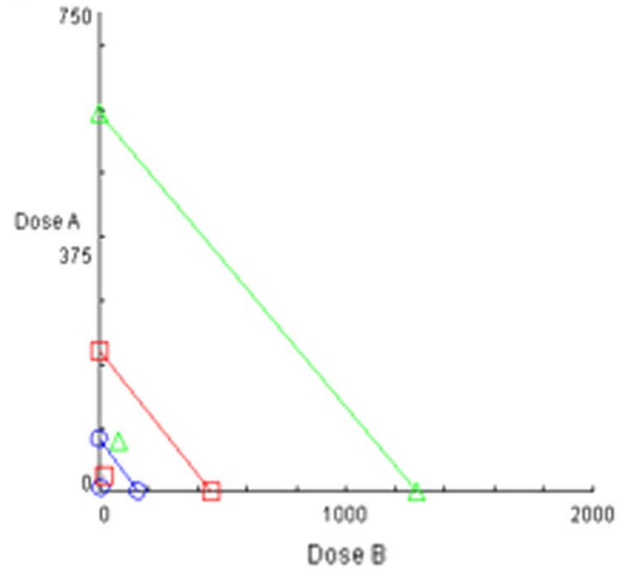

Fig. 4 Estimation of effect of drug combination using CompuSyn software a: Dose-Effect curve, b: Median- Effect plot, c: Combination index plot, d: Isobologram 
Table 3 Cytotoxicity study using C6 glioblastoma cell line

\begin{tabular}{lcccl}
\hline S. no & $\begin{array}{l}\text { Dose } \\
(\mu \mathrm{g} / \mathrm{ml})\end{array}$ & \multicolumn{3}{l}{ Percentage cell death } \\
\cline { 3 - 5 } & & $\mathrm{TMZ}$ & $\mathrm{NRG}$ & $\mathrm{TMZ}+\mathrm{NRG}$ \\
\hline 1. & 500 & 86.39 & 74.87 & 97.67 \\
2. & 250 & 73.06 & 60.22 & 90.11 \\
3. & 125 & 68.36 & 49.65 & 86.16 \\
4. & 62.5 & 46.19 & 22.76 & 78.83 \\
5. & 31.25 & 27.5 & 15.33 & 65.44 \\
6. & 15.63 & 14.52 & 9.59 & 49.17 \\
7. & 7.810 & 4.56 & 3.11 & 38.22 \\
$\mathrm{IC}_{50}$ values in $(\mu \mathrm{g} / \mathrm{ml})$ & 83.05 & 162.93 & 15.50 \\
\hline
\end{tabular}

Percentage cell death upon treatment with corresponding dose required as evaluated by SRB assay using C6 glioblastoma cells

Table 4 Interaction calculation using CompuSyn

\begin{tabular}{lccc}
\hline Parameters & TMZ group & NRG group & TMZ + NRG group \\
\hline "m" value & $1.11 \pm 0.06$ & $1.06 \pm 0.05$ & $1.06 \pm 0.05$ \\
"dm" value & 83.05 & 162.94 & 15.50 \\
"r" value & 0.99 & 0.99 & 0.98 \\
\hline
\end{tabular}

$\mathrm{m}, \mathrm{dm}$ and $\mathrm{r}$ values of the treatment groups calculated using CompuSyn

Table 5 Fraction affected with dose required and combination index value of drug combination (TMZ + NRG)

\begin{tabular}{llll}
\hline S. No. & Fraction affected & $\begin{array}{l}\text { Required dose }(\mu \mathrm{g} / \\
\mathrm{ml})\end{array}$ & $\begin{array}{l}\text { Combina- } \\
\text { tion index }\end{array}$ \\
\hline 1 & 0.5 & 15.50 & 0.14 \\
2 & 0.75 & 49.63 & 0.16 \\
3 & 0.85 & 97.35 & 0.18 \\
4 & 0.9 & 158.90 & 0.19 \\
5 & 0.95 & 350.65 & 0.21 \\
6 & 0.97 & 615.81 & 0.23 \\
\hline
\end{tabular}

Fraction affected with corresponding dose required along with combination index as calculated by CompuSyn software

\section{AO/EB staining for apoptosis/necrosis detection}

$\mathrm{AO} / \mathrm{EB}$ staining procedure revealed that the percentage of apoptotic cells significantly increased in case of TMZ + NRG combination treatment $(94.66 \pm 1.45)$ when compared to normal (3.66 \pm 0.33$)$, TMZ alone $(48.33 \pm 3.52)$ and NRG alone $(20.66 \pm 3.17)$ groups (Figs. 5 and 6$)$.

\section{Discussion}

In this study, the potential of naringin to inhibit PARP-1 responsible for $\mathrm{TMZ}$ resistance was evaluated using computational simulation and cell cytotoxicity assay. From the results obtained, it was inferred that the binding of naringin at the inhibitory site of PARP-1 was compatible, stable and strong. Combined evidences from both in silico and in vitro analysis showed the effectiveness of naringin in reversing TMZ resistance and to the best of our knowledge, this stands as a novel finding in the field of neuro-oncology.

XP docking result stands as proof of the potential establishment of strong interaction involving key amino acid residues of the target and naringin. The interaction diagram displayed under Table 2 reveals multiple non-covalent bonds launched which contributes to the stability and resilient binding. Backing this, calculated docking score and binding energy also depict the strong affinity. As quoted in previous literature, an RMSD value below $4 \AA$ is considered as good, since protein and ligand undergo minimal fluctuation (Hevener et al. 2009). In this case, stability was established soon after $15 \mathrm{~ns}$ and the number of interactions increased. Figure 2 illustrates the plot of ligand and protein contacts and interaction fraction. For the entire simulation duration of $20 \mathrm{~ns}$ average of 20 contacts between the ligand and protein can be observed with a maximum of 25 contacts and a minimum of 10 contacts. The majority of contacts were made by the residues ASP766, Asp770, His862, Gly863, SER864, Arg878, Tyr896, Tyr907 and Glu988 of the protein (hydrogen bond, water bridge, and hydrophobic interactions) with multiple hydroxy and oxy groups present in the ligand. Residues ARG885, Asn868, Gly888, Tyr889, Met890, Lys903and His909 were also observed to form a minor number of contacts during the simulation. An increase in RMSD (from 3.2 to $5.6 \AA ̊$ in protein and 1.6 to $3.2 \AA \AA$ in ligand) was observed after $10 \mathrm{~ns}$ which might be due to the formation of extra contacts with residue Ser864. Similarly, a slight increase in RMSD after $17.5 \mathrm{~ns}$ might be linked with the loss of contact with residue Gly888 and Glu988 or the formation of new contact with Tyr689. In comparison with co-crystallized PARP-1 inhibitor talazoparib, naringin showed an increase in the total number of interactions which includes all the key binding moieties. Naringin easily replaced Talazoparib from its binding site and formed more hydrogen bonds and pi-pi interactions when compared to Talazoparib. The presence of polyphenolic structure in naringin is the principal reason for complementary and multiple binding to the target, which is also in collateral with plenty of literature.(Alqahtani et al. 2019; Su et al. 2017).

Cell line studies were performed using C6 glioma cells as a proceeding step of validating computational 
Fig. 5 Detection of apoptosis/ necrosis by $\mathrm{AO} / \mathrm{EB}$ staining procedure a: Normal control, b: TMZ alone, $\mathbf{c}$ : NRG alone and $\mathbf{d}$ : TMZ + NRGN combination. Blue arrow head: live cells (green) and red arrow head: apoptotic cells (red)
Fig. 6 Effect of drug treatments on cell death as evaluated by $\mathrm{AO} / \mathrm{EB}$ staining procedure all the values are expressed as mean \pm SEM. ${ }^{\text {aa }} P<0.01$ vs Normal control, ${ }^{\text {aaa }} P<0.001$ vs Normal control, ${ }^{b b b} P<0.001$ vs TMZ, ${ }^{c c c} P<0.001$ vs NRG
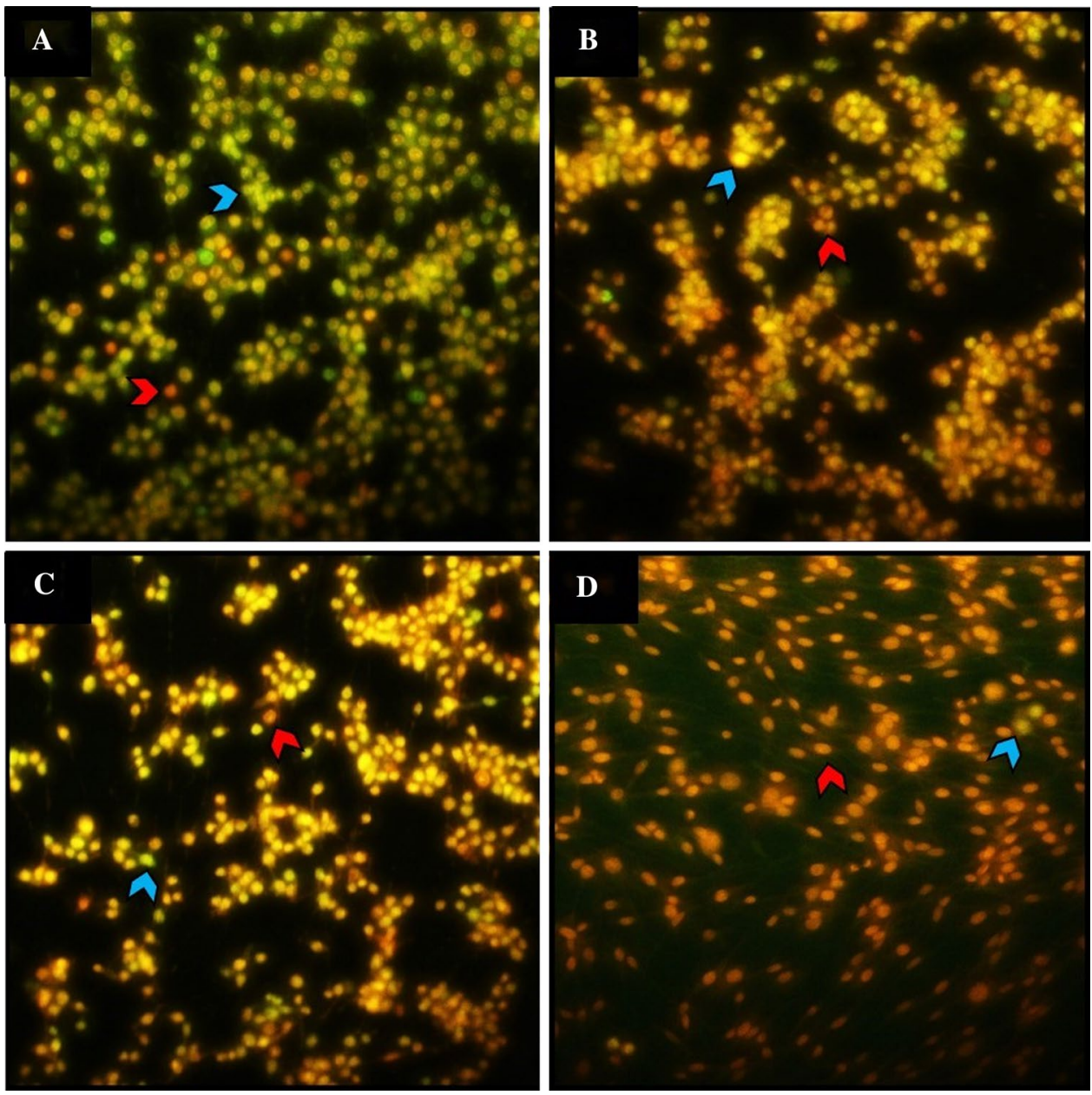

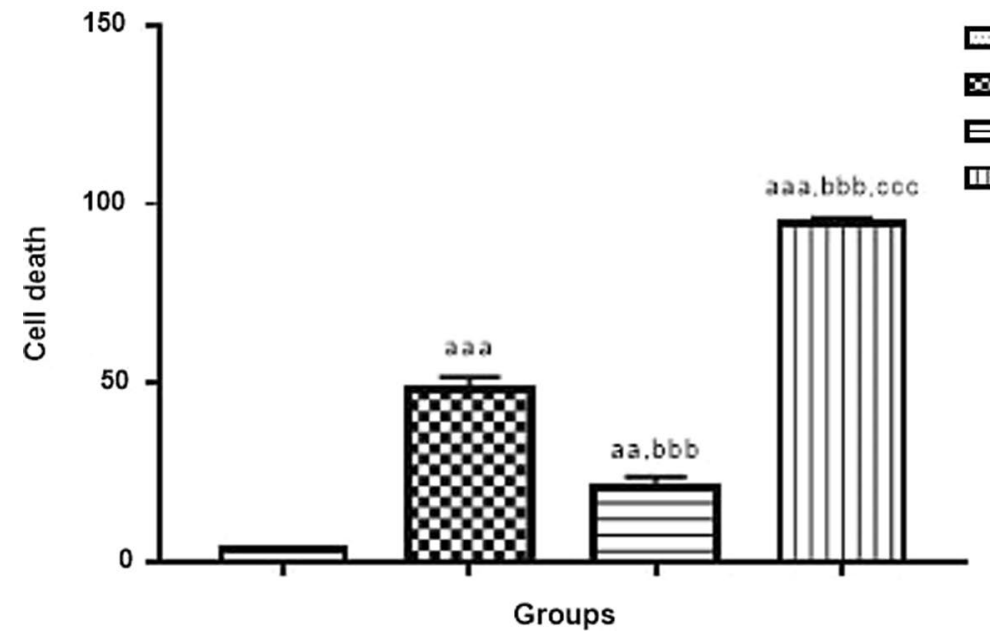

simulation. C6 glioma cell line was chosen for this study based on its expression profile regarding PARP-1. A study by Sevigny et. al. showed that the nuclear to cytoplasmic activity ratio of PARP-1 was highest in C6 glioma cells (Sevigny et al. 2003b). Significant lowering of $\mathrm{IC}_{50}$ dose when naringin was co-administered with $\mathrm{TMZ}$ at 1:1 ratio.
Overexpression of PARP in C6 cell lines upon treatment with methylating agents is one of the key factors in gaining the tag of TMZ-resistant cell line.(Sevigny et al. 2003a) This being the hypothesis, inhibition of PARP-1 by naringin favoured TMZ, with no major DNA repairing system to reverse the methylation. Hence $50 \%$ of the cell death 
had occurred with a lesser dose (TMZ + NRG) when compared to TMZ alone treatment. In accordance with the in silico results, this significant reduction in $\mathrm{IC}_{50}$ dose when given along with naringin indicates the effective inhibition of PARP-1 in the glioma cell lines, thereby resetting chemo-sensitivity to TMZ.

In order to reconfirm the type of effect seen when two drugs are given in combination, the acquired data were subjected to computational analysis using CompuSyn software (Chou 2010). Initially " $r$ " value was checked to verify the conformity of the data entered ( $>95$ ). Drug combination depicting synergism was highlighted in combination index plot and isobologram. An increase in cell death in accordance with the dose administered was plotted in the dose-effect plot. The lower red curve symbolizing naringin shows the least effectiveness against C6 glioma cells, followed by the temozolomide curve (blue). this effect is further supported by AO/EB staining procedure. A significant increase in the apoptotic cells in combination treatment reveals the effectiveness of naringin in reversing TMZ resistance. Being one of the most potent methylating agents, temozolomide exhibits lesser effectiveness in glioma due to the development of resistance. The presence of base excision repair system with mediating enzyme PARP-1 is postulated for the emergence of resistance. This ineffectiveness of temozolomide was overcome by adding naringin at the ratio of 1:1 ( $\pi$ et al. 2016). When combined, naringin lowers the activity of PARP-1, henceforth reversing temozolomide resistance and increasing its effectiveness in glioma as illustrated by the green curve in the dose-effect plot.

In addition to restoring chemo-sensitivity, PARP-1 inhibition by naringin might also help in glioma reduction, as recent evidences have shown that, inhibition of PARP-1 induces anti-angiogenic effects which might impact the tumor growth. Plenty of clinical trials with PARP-1 inhibitors in primary or secondary brain tumors showed cytotoxic effects in monotherapy especially in cases of BRCA (Breast Cancer gene) mutated tumors, which are defective in the homologous recombination (HR) repair (Sevigny et al. 2003b; Leonetti et al. 2012).

\section{Conclusion}

In conclusion, naringin, a bio-flavonoid, successfully reversed the chemo-resistance in C6 glioma cells when given in combination with TMZ, by inhibiting major alleged resistance mediator PARP-1, as initially evaluated using computational simulation method. This finding will open the arena for more phytochemicals to be considered for drug resistance reversal studies. The use of naturally obtained Phytomolecules in place of synthetic chemicals might lessen the occurrence of severe systemic side effects in cancer patients.
Further, studies in pre-clinical animal glioma models of TMZ resistance are required to validate the above-mentioned findings.

Supplementary Information The online version contains supplementary material available at https://doi.org/10.1007/s11696-021-01700-0.

Acknowledgements We thank Manipal Academy of Higher Education for funding (Intramural Scheme) and infrastructural Support.

Authors' contribution VR, KN, CMR and NK involved in conceptualization and design of experiment; VR, SPC, SM, RJAV and NK participated in collection of data; VR, SPC, SM, CMR and NK involved in data analysis and interpretation; VR, RJAV and NK participated in manuscript writing.

Funding Open access funding provided by Manipal Academy of Higher Education, Manipal. We thank Manipal Academy of Higher Education for providing Intramural funding for the completion of the project. The Funding was granted to Ms R.J.A. Vibhavari (190600136) under the Intramural funding scheme of MAHE, Manipal.

\section{Declarations}

Conflict of interest We declare no conflict of interest.

Consent for publication All the authors give their consent for the publication of this article.

Open Access This article is licensed under a Creative Commons Attribution 4.0 International License, which permits use, sharing, adaptation, distribution and reproduction in any medium or format, as long as you give appropriate credit to the original author(s) and the source, provide a link to the Creative Commons licence, and indicate if changes were made. The images or other third party material in this article are included in the article's Creative Commons licence, unless indicated otherwise in a credit line to the material. If material is not included in the article's Creative Commons licence and your intended use is not permitted by statutory regulation or exceeds the permitted use, you will need to obtain permission directly from the copyright holder. To view a copy of this licence, visit http://creativecommons.org/licenses/by/4.0/.

\section{References}

Alqahtani S, Welton K, Gius JP, Elmegerhi S, Kato TA (2019) The effect of green and black tea polyphenols on BRCA2 deficient Chinese hamster cells by synthetic lethality through PARP inhibition. Int J Mol Sci 20(6):1274

Aslantürk ÖS (2018) In vitro cytotoxicity and cell viability assays: principles, advantages, and disadvantages, vol 2. InTech, London

Berman HM, Westbrook J, Feng Z, Gilliland G, Bhat TN, Weissig H, Shindyalov IN, Bourne PE (2000) The protein data bank. Nucl Acids Res 28(1):235-242

Chou T-C (2010) Drug combination studies and their synergy quantification using the Chou-Talalay method. Cancer Res 70(2):440-446

Chou T, Martin N (2005) CompuSyn for drug combinations: PC software and user's guide: a computer program for quantitation of synergism and antagonism in drug combinations, and the determination of IC50 and ED50 and LD50 values. ComboSyn, Paramus. https://www.combosyn.com/. Accessed 6 June 2020 
Cloughesy TF, Cavenee WK, Mischel PS (2014) Glioblastoma: from molecular pathology to targeted treatment. Annu Rev Pathol 9:1-25

Elokely KM, Doerksen RJ (2013) Docking challenge: protein sampling and molecular docking performance. J Chem Inf Model 53(8):1934-1945

Franzese E, Centonze S, Diana A, Carlino F, Guerrera LP, Di Napoli M, De Vita F, Pignata S, Ciardiello F, Orditura M (2019) PARP inhibitors in ovarian cancer. Cancer Treat Rev 73:1-9

Goldsmith MA, Carter SK (1974) Glioblastoma multiforme-A review of therapy. Cancer Treat Rev 1(2):153-165

Gupta SK, Smith EJ, Mladek AC, Tian S, Decker PA, Kizilbash SH, Kitange GJ, Sarkaria JN (2019) PARP inhibitors for sensitization of alkylation chemotherapy in glioblastoma: impact of blood-brain barrier and molecular heterogeneity. Front Oncol 8:670

Hevener KE, Zhao W, Ball DM, Babaoglu K, Qi J, White SW, Lee RE (2009) Validation of molecular docking programs for virtual screening against dihydropteroate synthase. J Chem Inf Model 49(2):444-460

Higuchi F, Nagashima H, Ning J, Koerner MV, Wakimoto H, Cahill DP (2020) Restoration of temozolomide sensitivity by poly (ADPRibose) polymerase inhibitors in mismatch repair deficient glioblastoma is independent of base excision repair. Clin Cancer Res 26:1690-1699

Ignjatović MM, Caldararu O, Dong G, Munoz-Gutierrez C, AdasmeCarreno F, Ryde U (2016) Binding-affinity predictions of HSP90 in the D3R grand challenge 2015 with docking, MM/GBSA, $\mathrm{QM} / \mathrm{MM}$, and free-energy simulations. J Comput Aided Mol Des 30(9):707-730

Ivanova L, Tammiku-Taul J, García-Sosa AT, Sidorova Y, Saarma M, Karelson M (2018) Molecular dynamics simulations of the interactions between glial cell line-derived neurotrophic factor family receptor GFR $\alpha 1$ and small-molecule ligands. ACS Omega 3(9):11407-11414

Johannessen T-CA, Bjerkvig R (2012) Molecular mechanisms of temozolomide resistance in glioblastoma multiforme. Expert Rev Anticancer Ther 12(5):635-642

Jourdan PS, Weiler EW, Mansell RL (1985) Naringin levels in citrus tissues: I. Comparison of different antibodies and tracers for the radioimmunossay of naringin. Plant Physiol 77(4):896-902

Kumar SR, Ramli M, Suhana E, Nasir A, Alimah N, Ismail NHM, Fahami M, Azlina N (2019) Preventive effect of naringin on metabolic syndrome and its mechanism of action: a systematic review. Evid Based Complement Alternat Med 2019:1-11

Lee SY (2016) Temozolomide resistance in glioblastoma multiforme. Genes Dis 3(3): 198-210

Leonetti C, Biroccio A, Graziani G, Tentori L (2012) Targeted therapy for brain tumours: role of PARP inhibitors. Curr Cancer Drug Targ 12(3):218-236. https://doi.org/10.2174/156800912799277 403

Messaoudi K, Clavreul A, Lagarce F (2015) Toward an effective strategy in glioblastoma treatment. Part I: resistance mechanisms and strategies to overcome resistance of glioblastoma to temozolomide. Drug Discov Today 20(7):899-905

Newlands E, Stevens M, Wedge S, Wheelhouse RT, Brock C (1997) Temozolomide: a review of its discovery, chemical properties, pre-clinical development and clinical trials. Cancer Treat Rev 23(1):35-61

Nijveldt RJ, Van Nood E, Van Hoorn DE, Boelens PG, Van Norren K, Van Leeuwen PA (2001) Flavonoids: a review of probable mechanisms of action and potential applications. Am J Clin Nutr 74(4):418-425

Rathi E, Kumar A, Kini SG (2019) Molecular dynamics guided insight, binding free energy calculations and pharmacophore-based virtual screening for the identification of potential VEGFR2 inhibitors. J Recept Sign Transduct Res 39(5-6):415-433

Salehi B, Fokou PVT, Sharifi-Rad M, Zucca P, Pezzani R, Martins N, Sharifi-Rad J (2019) The therapeutic potential of naringenin: a review of clinical trials. Pharmaceuticals 12(1):11

Sevigny MB, Silva JM, Lan W-C, Alano CC, Swanson RA (2003a) Expression and activity of poly (ADP-ribose) glycohydrolase in cultured astrocytes, neurons, and C6 glioma cells. Brain Res Mol Brain Res 117(2):213-220

Sevigny MB, Silva JM, Lan WC, Alano CC, Swanson RA (2003b) Expression and activity of poly(ADP-ribose) glycohydrolase in cultured astrocytes, neurons, and C6 glioma cells. Brain Res Mol Brain Res 117(2):213-220. https://doi.org/10.1016/s0169$328 \mathrm{x}(03) 00325-5$

Su C, Haskins AH, Omata C, Aizawa Y, Kato TA (2017) PARP inhibition by flavonoids induced selective cell killing to BRCA2-deficient cells. Pharmaceuticals 10(4):80

Tiwari A, Kutty NG, Kumar N, Chaudhary A, Raj PV, Shenoy R, Rao CM (2016) Synthesis and evaluation of selected 1, 3, 4-oxadiazole derivatives for in vitro cytotoxicity and in vivo anti-tumor activity. Cytotechnology 68(6):2553-2565

Trivedi RN, Almeida KH, Fornsaglio JL, Schamus S, Sobol RW (2005) The role of base excision repair in the sensitivity and resistance to temozolomide-mediated cell death. Cancer Res 65(14):6394 6400. https://doi.org/10.1158/0008-5472.can-05-0715

Zhang J, Stevens MF, Bradshaw TD (2012) Temozolomide: mechanisms of action, repair and resistance. Curr Mol Pharmacol 5(1):102-114. https://doi.org/10.2174/1874467211205010102

Zhang N, Fu J-N, Chou T-C (2016) Synergistic combination of microtubule targeting anticancer fludelone with cytoprotective panaxytriol derived from panax ginseng against MX-1 cells in vitro: experimental design and data analysis using the combination index method. Am J Cancer Res 6(1):97

Publisher's Note Springer Nature remains neutral with regard to jurisdictional claims in published maps and institutional affiliations. 